\title{
Reliability Impact on Planetary Robotic Missions
}

\author{
David Asikin and John M. Dolan
}

\begin{abstract}
In the mobile robotics literature, there is little formal discussion of reliability and failure. Moreover, current work focuses more on the assessment of existing robots. In contrast, our work predicts the impact on reliability on robotic missions. In our previous work, we presented a quantitative analysis to predict the probability of robot failure during a mission and use this to compare the performance of different robot team configurations. In order to comprehensively characterize robot failure, we proposed a taxonomy system which divides planetary robotic missions into three classes and showed how the taxonomy can be used as a framework to explore the reliability characteristics of each mission class. In this paper, we define and simulate common mission scenarios for each class in the taxonomy system and attempt to extract general reliability trends and mission characteristics for given robot and environment parameters. Our results show that, for comparable mission scopes with a fixed budget, exploration-type missions have maximum mission success probability for smaller team sizes than is the case for construction-type missions.
\end{abstract}

\section{INTRODUCTION}

$\mathrm{T}_{\mathrm{c}}^{\mathrm{H}}$ HE current design of NASA planetary robots puts high emphasis on robot reliability. This extreme reliability has enabled robots to function far beyond their expected lifetime: the Mars Pathfinder Sojourner Rover operated 83 days, 12 times its expected lifetime of seven days [3]; the Mars Exploration Rovers (MER) have been operating for more than 6 years, rather than the intended 90 days. This reliability comes at a high cost, both in initial development costs and in the ongoing operational costs for their mission extensions. The need to reduce robot development costs has been one of the major problems in planning in-situ planetary missions [1]. Fiscal problems have delayed some missions: the Mars Science Laboratory was given the highest priority in NASA's Mars Program of 2002, but then delayed in the 2006 plan as a result of cost constraints [2].

Cost reductions can be achieved if overly reliable components are exchanged for ones more in line with mission requirements. For this, tradeoff analysis between component reliability, mission risk, and cost is needed. We introduced a quantitative methodology to perform such analysis in

Manuscript received February 28, 2010. This research was funded by the National Aeronautics and Space Administration (NASA) under award NNX07AV71G S01 in the Advanced Intelligent Systems Research (AISR) program.

D. Asikin is with the Robotics Institute, Carnegie Mellon University, Pittsburgh, PA, 15213-3890 USA (e-mail: dasikin@cs.cmu.edu).

J. M. Dolan is with the Robotics Institute, Carnegie Mellon University, Pittsburgh, PA, 15213-3890 USA (e-mail: jmd@cs.cmu.edu). previous work [4], [5], but the approach was limited to a small number of examples. In order to capture the reliability characteristics of planetary robotic missions, it is necessary to comprehensively study the mission space. Our approach is therefore to use a taxonomy that will allow examination of the full range of the mission space in a methodical manner, given the vast diversity of planetary robotic missions.

Several taxonomies for robot teams and robot tasks/missions have been proposed. [15] classified multirobot teams in terms of team size and composition, communications, and processing capability. [16] classified multirobot tasks in terms of time, energy, robot movement and capabilities. [17] categorized robot tasks by the amount and type of human-robot interaction involved. [18] used human operator function to classify UAV missions. [19] broke down intelligent robotics in terms of technologies and functionalities. While the proposed taxonomies mainly focus on robot capabilities and coordination, we hypothesize that the amount of time spent on mission tasks coupled with the reliability of robot components used in respective tasks is most determinative of the reliability characteristics of a mission. We therefore analyzed the typical tasks involved in mission instances proposed in the NASA Roadmap for the exploration of the Solar System over the next 30 years [7] and the Mars Exploration Program [8], and classified robot missions with respect to the time proportions of the mission tasks [6]. In [6], we also devised a methodology to optimize mission success rate and help mission designers estimate the appropriate robot team size and module reliability under a budget limitation in the planning stage.

Extending our work in that paper, here we propose mission completion time as one of the parameters to be considered in the optimization. More importantly, we fully define and stochastically simulate common mission scenarios for the three mission classes in the taxonomy. We analyze the time proportion of tasks involved in each class and observe dominant mission parameters in determining the stability of the time proportions. Finally, we analyze the performance of a given robot configuration in three mission classes and observe the reliability characteristics in each case to seek a general trend in planetary robotic missions.

\section{ROBOT MISSION TAXONOMY}

Based on a comprehensive survey of NASA in-situ planetary robotic missions proposed in the Solar System Exploration Roadmap (SSER) and Mars Exploration 
Program (MEP), we identified several fundamental "Basic Activities" which can be combined in different time proportions to form any mission. We propose that missions can be formed using the following nine basic activities: Traverse, Subsurface Access, Instrument Deployment, Sampling, Assembly, Communication, Sample Analysis, Recharging, Idling. Missions with similar sets of basic activities are close in nature and can be grouped together. Using this methodology, we categorize planetary robotic missions into three classes [6]: Search and Exploration (S\&E), Sample Acquisition and Composition Analysis (SA\&CA), and Construction. Mission instances in a given class have a distinguishing time proportion of basic activities (see section V-A). Though these proportions are not absolutely fixed, they fall into a range such that the character of a mission significantly changes only when the proportions exceed that range.

\section{Mission Scenarios And Environment Model}

We use the taxonomy as a framework for exploring the reliability characteristics and design tradeoffs in each mission class. We examine each mission class with a representative mission scenario allowing variation of mission parameters via stochastic simulation using a state transition diagram wherein each state corresponds to a basic activity. Using the methodology previously used to simulate the Construction mission class [6], here we define the general scenarios and the corresponding state transition diagrams for the remaining two mission classes (S\&E and SA\&CA).

\section{A. Search and Exploration Class}

For the S\&E Class, we consider a general scenario in a planetary environment to explore a bounded square area and map it using a team of robots where each robot works independently from the others. The area is partitioned into uniform cells with adjustable resolution according to the robot sensing radius. Each cell is then represented by a node. The mission is considered a success when all nodes are explored. The robots divide the S\&E task such that each node is only visited once. We experimented using the path planning algorithm introduced in [9], as well as a simple lawnmower pattern. If a robot fails, a spare robot (if available) is deployed from the starting location and new paths are calculated for each robot. We include a daily communication link between each robot and the command center on earth for robot coordination purposes and status updates. We consider energy limitations in the mission such that the robots need to generate energy through the use of solar panels daily. The robots also minimize the energy expended by entering the sleep/idle mode to wait for the sun if the battery is below an acceptable level. The availability of the sun and its duration are randomized to simulate uncertainty (e.g. dust, shadowed terrain, etc.) in battery recharging.

Making appropriate inferences from the on-line MER log

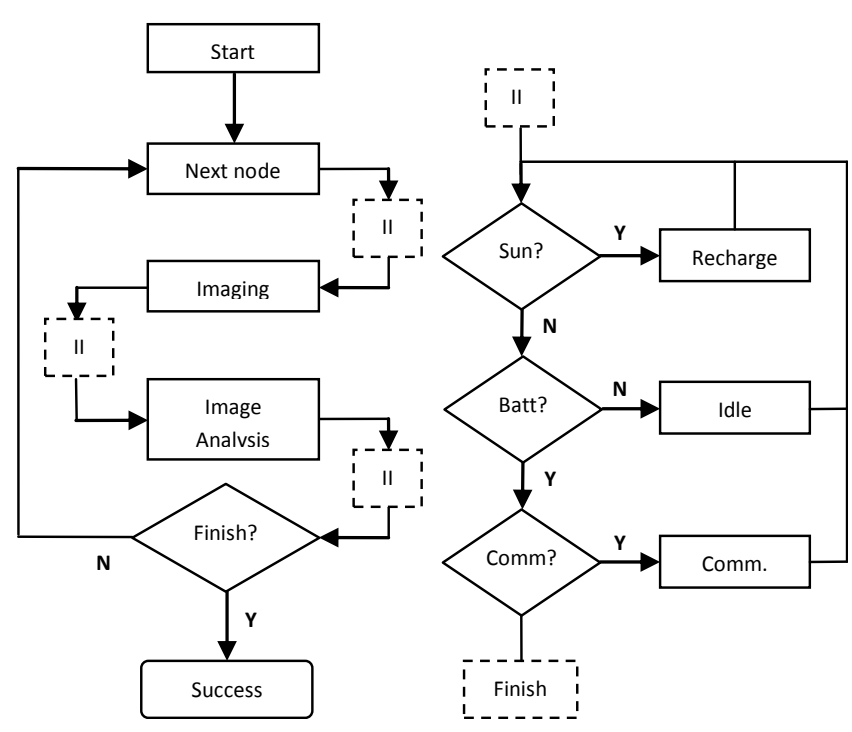

Fig. 1. State Transition Diagram for Search and Exploration mission class generated from the mission scenario described in subsection III.A.

[10], we modeled the flow of the simulation as closely as possible after the real MER routines. The mission flow is defined as the following 3 steps:

1. Traverse to an assigned node

2. Constantly capture images/map the area on the way

3 . Analyze the images

Repeat $1-3$ until all nodes are visited

Do $\mathrm{a}-\mathrm{c}$ in between the steps:

a. Replenish battery using solar panel whenever the sun is available

b. Communicate with Earth whenever the communication link is available

c. Activate sleep mode when the battery is low

The state diagram of the S\&E mission class is shown in Figure 1 .

\section{B. Sample Acquisition and Composition Analysis Class}

In a similar manner, here we define a general SA\&CA mission scenario. For this mission class, a team of robots in a planetary environment visits and collects rock samples from several sites of interest through the use of manipulator drills. , We use the same path planning algorithm and sampling environment as in the S\&E Class. In this case, however, a rock sample needs to be collected from each node in the uniform grid. However, there is a major underlying difference from the S\&E Class in the nature of the tasks involved. The S\&E Class emphasizes mapping a region of interest, so it requires heavy imaging and analysis of the images. In contrast, the SA\&CA Class requires minimal imaging. The mission flow is defined as the following 4 steps:

1. Traverse to an assigned node

2. Drill at the node

3. Collect a soil sample 
4. Analyze the sample

Repeat $1-4$ until all nodes are visited

Do $\mathrm{a}-\mathrm{c}$ in between the steps:

a. Replenish battery whenever the sun is available

b. Communicate with Earth when comm. link is available

c. Activate sleep mode when the battery is low

The mission is considered successful when samples from all nodes are collected. The state transition diagram for this mission class is generated in a similar manner to the other mission classes and simulated stochastically according to the methodology introduced in [5].

\section{ROBOTS AND COMPONENTS}

For the purposes of this analysis, we assume that the robots on the team are homogeneous. We use the same robot specifications when simulating the three different mission classes in order to create a baseline by which we can compare the reliability characteristics of the mission classes.

The robots are considered to be made up of several subsystems that are independent from the standpoint of

TABLE I

ROBOT SUBSYSTEMS AND RELIABILITIES

\begin{tabular}{|c|c|}
\hline Subsystem & MTTF (h) \\
\hline Power & 4202 \\
\hline Computation \& Sensing & 4769 \\
\hline Mobility & 19724 \\
\hline Communications & 11876 \\
\hline Manipulator & 13793 \\
\hline
\end{tabular}

reliability. The subsystem reliabilities listed in Table I are derived from [4].

We model the robots as closely as possible on the Mars Exploration Rovers (MER), setting the weight to $174 \mathrm{~kg}$ [11] and equipping them with two $7.15-\mathrm{kg}$ lithium ion batteries $(150 \mathrm{~W}-\mathrm{h} / \mathrm{kg})$ for energy storage. The solar panels on the robots generate 700 watt-hours per day on average.

TABLE II

POWER CONSUMPTION MODEL

\begin{tabular}{|c|c|}
\hline Basic Activity & Power Consumption \\
\hline Traverse & $100 \mathrm{~W}$ \\
\hline Imaging / Mapping & $29 \mathrm{~W}$ \\
\hline Soil Sampling & $52 \mathrm{~W}$ \\
\hline Subsurface Access & $55 \mathrm{~W}$ \\
\hline Sample / Image Analysis & $19 \mathrm{~W}$ \\
\hline Communication & $74 \mathrm{~W}$ \\
\hline Idling & $10 \mathrm{~W}$ \\
\hline
\end{tabular}

The power consumption model used in the simulation is derived from [12] and listed in Table II. Based on [13], the robot speed is set to a constant $0.01 \mathrm{~m} / \mathrm{s}$ throughout the mission. Inferring from [14], we also introduce a limitation on terrain visibility causing the robot to stop and observe its surroundings after it has traversed a distance equal to its sensing radius. For the SA\&CA mission scenario, each robot on the team is assumed to have an average drilling time of 3 hours per site [20].

\section{RESULTS}

We calculate the probability of the baseline robot configuration's successful completion of the mission scenarios described in previous sections. The simulation is repeated many times with the average score of all trials giving the overall probability of mission completion (PoMC).

To comprehensively capture the reliability characteristics of the three mission classes, for each mission scenario, we vary the environment model and observe the resulting PoMC. We also record the time spent on each basic activity in a mission and analyze the resulting time proportions. The input parameters to be varied are the following:

- Number of robots and spare robots

- Size of the area to be explored / from which rock samples are to be collected

- Reliability of the robot modules

- Robot sensing radius

Given the hyper-dimensionality of the model, we simplify the analysis by varying only one variable at a time and fixing the rest, then looking at the relationship between the varied variable and the PoMC, as well as the time proportion of the basic activities. We set the baseline variables as shown in Table III and then increment one variable along the $\mathrm{x}$-axis from the minimum to the maximum expected value.

TABLE III

BASELINE CONSTANTS USED IN THE SIMULATION

\begin{tabular}{|c|c|c|}
\hline Variable & Baseline Value & Range \\
\hline \#Nodes & 30 & $1-100$ \\
\hline Area size & $30 \times 30 \mathrm{~m}$ & $30 \times 30 \mathrm{~m}-130 \times 130 \mathrm{~m}$ \\
\hline \#Robots & 2 & $1-10$ \\
\hline \#Spare robots & 0 & $0-5$ \\
\hline \%MTTF & $100 \%$ & $0-100 \%$ \\
\hline Sensing radius & $5 \mathrm{~m}$ & $1-5 \mathrm{~m}$ \\
\hline
\end{tabular}

Finally, we consider budget constraints in the simulation. We adopt a general relationship of module reliability and cost, where cost is an exponential function of component reliability. Using the cost model and approach introduced in [6], we analyze the optimum robot configuration in terms of robot team size and component reliability for each mission scenario for a given set of input parameters. 


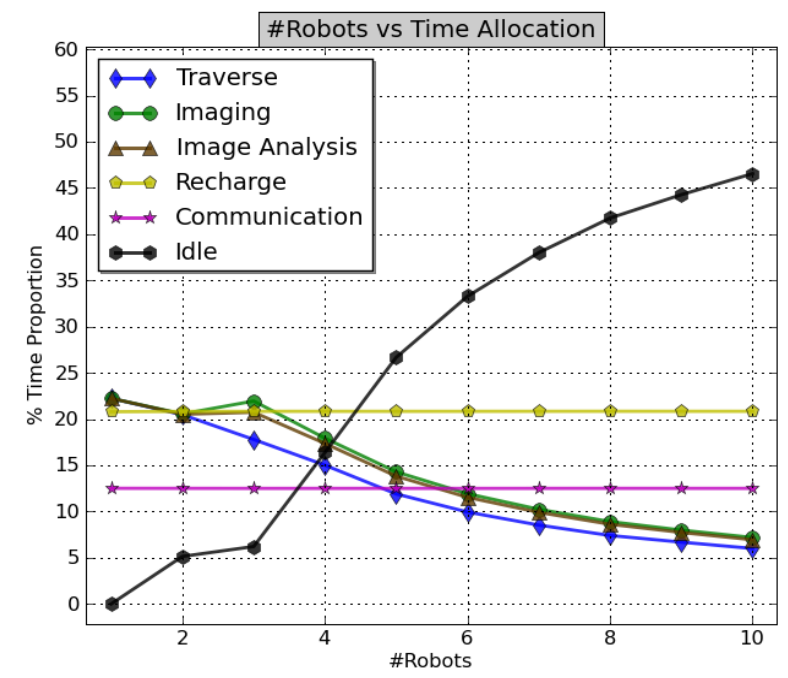

Fig. 2. Varying number of robots in Search and Exploration Class.

\section{A. Sensitivity Analysis of the Mission Parameters}

For the Construction class, the resulting time proportion of basic activities is relatively stable under a reasonable range of mission parameters [6]. The S\&E and SA\&CA class show the same behavior for the majority of the input parameters, where the approximate resulting time proportion in Table IV can be obtained when the baseline constants (see Table III) are used under the specified range. This does not hold true for certain parameters discussed in the following.

The sensitivity analysis of the result shows that the time proportion of the basic activities for the S\&E class and SA\&CA class are largely dependent on the number of robots used in the mission. This agrees with our previous result for the Construction class in [6]. Increasing the number of robots causes causes each robot to be allocated fewer nodes to explore or collect samples from, thus decreasing the required amount of traverse, mapping/imaging, and analysis of the images (see Figure 2).

In contrast to the Construction class, the distance traveled by the robots is no longer a major factor in determining the time proportions for the S\&E and SA\&CA classes. The Construction class requires robots to carry modules from a module depot to the construction sites and assemble them, so increasing the size of the environment directly increases the relative proportion of Traverse to the remaining basic activities. However, due to the nature of the mission, increasing the size of the environment in the S\&E and SA\&CA classes only proportionally increases the area the robots need to explore or collect samples from, hence the stability of the time proportion regardless of the traveled distance (see Figure 3).

Table IV shows the time proportions of the basic activities of the three mission classes. Most of the Construction class mission time $(47 \% \pm 2 \%)$ is spent on assembling the modules, whereas the S\&E class has a balanced basic activity

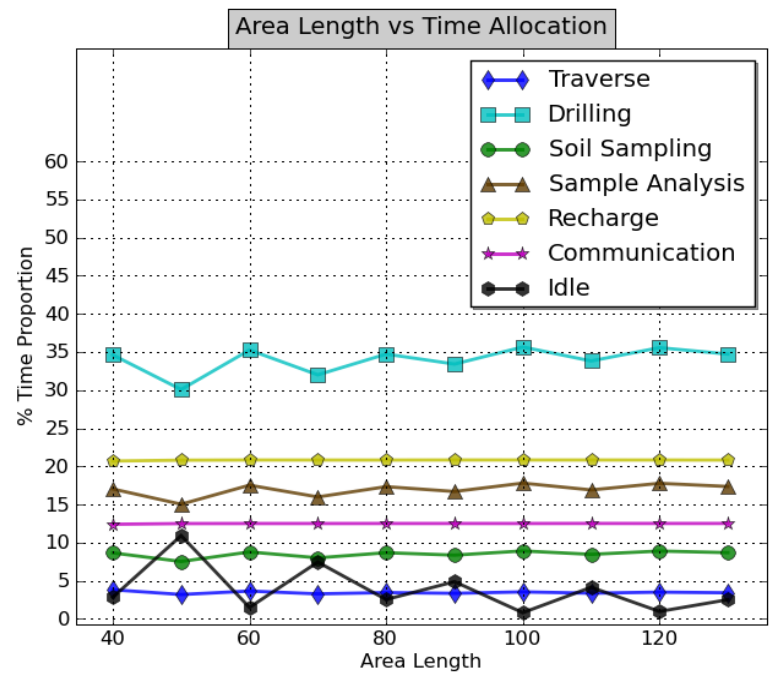

Fig. 3. Varying area length in Sample Acquisition and Composition Analysis Class.

proportion between Traverse, Imaging, and Image Analysis $(21 \% \pm 2 \%$ each), which are the core tasks for exploration type of missions. The SA\&CA class has a time proportion characteristic somewhat in between the other two classes. It has a small amount of Traverse and more time spent on using the manipulator (e.g. Drilling) as in the Construction class. This is somewhat counterintuitive at first, because the SA\&CA mission collects soil samples from the same area size as S\&E class, so it seems the amount of Traverse should be similar. However, under the baseline constants, the time spent on drilling each node far outweighs the time required to traverse from one node to the other, hence the increased Drilling time proportion.

TABLE IV

TIME PROPORTION FOR THE 3 MISSION ClASSES

\begin{tabular}{|c|c|c|c|}
\hline Basic Activities & $\begin{array}{c}\mathrm{C} \\
( \pm 2 \%)\end{array}$ & $\begin{array}{l}\text { S\&E } \\
( \pm 2 \%)\end{array}$ & $\begin{array}{c}\text { SA\&CA } \\
( \pm 4 \%)\end{array}$ \\
\hline Traverse & $4 \%$ & $21 \%$ & $4 \%$ \\
\hline $\begin{array}{l}\text { Instrument } \\
\text { Deployment }\end{array}$ & $21 \%$ & $0 \%$ & $0 \%$ \\
\hline Module Assembling & $47 \%$ & $0 \%$ & $0 \%$ \\
\hline $\begin{array}{c}\text { Imaging / } \\
\text { Soil Sampling }\end{array}$ & $0 \%$ & $21 \%$ & $9 \%$ \\
\hline Drilling & $0 \%$ & $0 \%$ & $32 \%$ \\
\hline $\begin{array}{c}\text { Image/Sample } \\
\text { Analysis }\end{array}$ & $0 \%$ & $21 \%$ & $17 \%$ \\
\hline Communication & $17 \%$ & $12 \%$ & $13 \%$ \\
\hline Recharging & $11 \%$ & $21 \%$ & $21 \%$ \\
\hline Sleep/Idle & $<1 \%$ & $<5 \%$ & $<5 \%$ \\
\hline
\end{tabular}

\section{B. Comparative Performance Analysis of Mission Classes}

We compared the PoMC of a baseline team of 2 robots completing each mission scenario of constructing 50 sites on a region $25 \mathrm{~m}$ apart from the module depot, mapping 50 nodes on the area with the size of $30 \times 30 \mathrm{~m}$, and, collecting samples 
from 50 nodes inside $30 \times 30 \mathrm{~m}$ area. We obtain differing PoMC values of $60 \%, 72 \%$, and, $96 \%$, respectively. The result shows that the Construction class has the lowest mission success rate if performed by the given robot configuration.

Comparing the performance of the same robot configuration across different mission scenarios is especially useful when the mission designer does not have the ability to change the robot specification (e.g. the mission has to be performed by the available or surviving robots). Thus, from the simple analysis we can determine the most suitable mission for the current robot team, which is to collect samples from 50 nodes, as it gives the best chance of completion compared to the other mission types.

Alternatively, mission designers can fix the PoMC and ask how much can be accomplished by different missions. For example, with $60 \%$ PoMC, the same team of robots can either construct 50 sites or explore 61 sites. Coupled with some other measure (e.g. scientific gain) of the relative per-site value of performing one or the other mission, this could allow a designer to compare expected value of different missions.

\section{Optimizing PoMC and Mission Time with Fixed Cost}

Considering a mission budget constraint, we can seek the optimum robot configuration in terms of robot team size and module reliability that maximizes the mission success rate for the S\&E class and SA\&CA classes using the cost model introduced in [6]:

$$
C(R)=2 \mathrm{e}^{1.61 \mathrm{R}+2.3},
$$

where $\mathrm{R}$ is the percentage of component reliability compared to the baseline model. Note that the cost model serves as an example and can be replaced with any cost model a mission designer has.

In Figure 4, using the baseline values listed in Table III as the input variables, we plot several tradeoff relations between component reliability (\%MTTF) and mission success rate (PoMC) for different robot team sizes. We also fit curves to these points, allowing the \%MTTF to be estimated for intermediate points without running additional simulations. The black horizontal line shows the PoMC for the baseline configuration (1 robot with $100 \%$ of the baseline component reliability). Based on the cost model (Equation 1), we are able to compute the maximum achievable component reliability (\%MTTF) under the budget constraint for each team size. This is shown as dashed vertical lines.

The intersection between the dashed vertical lines and the PoMC curve (a function of \%MTTF) then gives the maximum achievable PoMC for each team configuration (using the maximum achievable \%MTTF) given the budget constraint. The intersections are shown as stars on the dashed curve. For a S\&E mission with area $40 \mathrm{~m} \times 40 \mathrm{~m}$, the configuration of 2 robots with $57.1 \%$ MTTF (of the baseline MTTF in Table I) gives the highest mission success rate, which is $84.9 \%$ PoMC.

Here we extend our work in [6] by also considering mission duration as a part of the optimization problem. The cost model implemented in the simulation only considers robot development cost. Mission designers might have different priorities, such as limited mission time to cut the mission operational cost. In that case, configurations with a higher number of robots and less module reliability might be preferable to reduce the time required to complete the task.

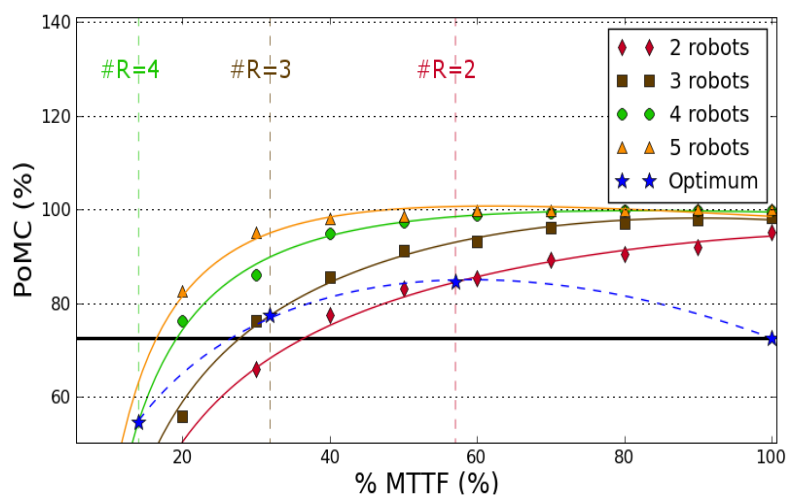

Fig. 4. Optimum robot configuration for Search and Exploration mission scenario when area size $=40 \mathrm{~m} \times 40 \mathrm{~m}$.

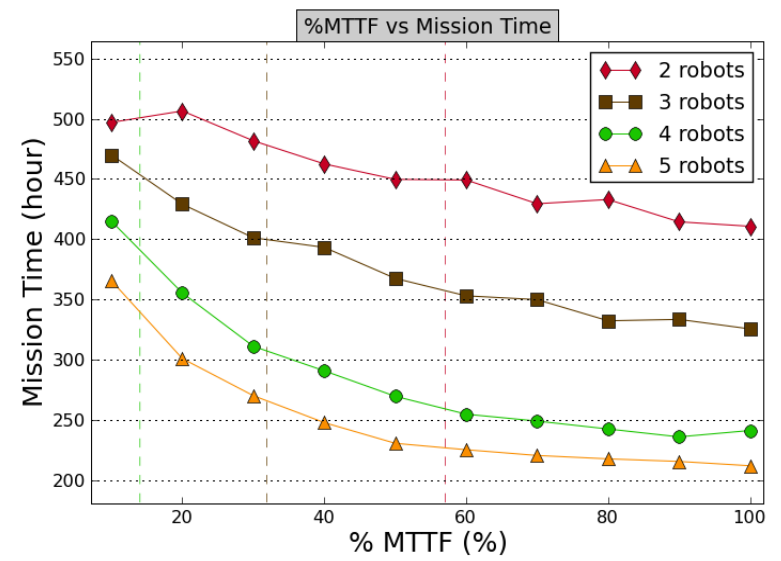

Fig. 5. Mission time for Search and Exploration mission scenario when area size $=40 \mathrm{~m} \times 40 \mathrm{~m}$.

Figure 5 shows the time required to complete the same mission for each robot team size. 2 robots with $57.1 \%$ MTTF require 450 hours (intersection of vertical dashed line with the mission time line for 2 robots), whereas 3 robots with $31.9 \%$ MTTF only require 400 hours to complete the mission. Thus, the latter might be preferable in some cases, with only a slight reduction of PoMC (approximately 7.3\%, see Figure 4). The solution can also be combined by comparing it with the PoMC for the baseline configuration (73\%) to get the most appropriate robot configuration that 
delivers better PoMC than the baseline and yet satisfies the mission time limit.

\section{Mission Duration-Based Robot Design Policy}

In [6] we showed that the optimum robot configuration (number of robots coupled with specific component reliability) changes as the mission duration is prolonged. Analyzing and comparing this behavior across three mission classes is crucial in understanding the reliability characteristics of each mission class.

For relatively short missions, the PoMC gain per robot number increase is likely to be larger than the loss of PoMC per component reliability decrease. Thus, under a budget constraint, it is more beneficial to use more robots with decreased module quality in short missions. And, finally, there is a turning point where the PoMC gain per robot number increase is equal to the PoMC gain per component reliability increase as mission duration is extended.

Here we gradually increased the mission duration for the three mission classes and observed where the turning point (optimum configuration change from 3 robots to 2 robots) occurs. We discovered that the turning point occurs the earliest in the S\&E class (270 hours, 92\% PoMC), followed by SA\&CA class (500 hours, $82 \%$ PoMC) and Construction class ( 820 hours, $66 \%$ PoMC).

The order of occurrence of the turning points is, of course, dependent on the robot and mission parameters. However, using robot specifications modeled after the MER and the cost model (Equation 1) which is an exponential function of component reliability, the S\&E class prefers a smaller number of higher-quality robots, whereas the Construction class is better performed by a larger number of lower-reliability robots.

This methodology is applicable as a metric in determining robot design policy (e.g. large team vs. small team compatibility on different mission classes) in the conceptual mission planning stage.

\section{CONCLUSiON}

This paper extends our work in [6] by detailing common mission scenarios for the planetary robotic mission taxonomy. Simulating the mission scenarios, we discovered that small teams of robots with highly reliable components have better performance in executing Exploration type of missions, whereas large team of robots with low component reliability is preferable in Construction missions for the given robot and mission specification. We also extended our previous work by considering mission duration when optimizing PoMC with respect to robot team size, component reliability, and cost. Finally, we confirmed the stability of the time proportion for each mission class and analyzed the shifting behavior of their optimum robot configuration by extending the mission duration.
In future work, we will perform reliability analysis of each mission class on the module level to seek connection between the time proportion of the basic activities and PoMC of the missions, as well as identification of critical modules to the mission. In addition, we intend to introduce heterogeneous robots and repair capability in the case of robot failure. Another interesting area is the consideration of partial failure in the simulation.

\section{REFERENCES}

[1] Green, J. L. 2008. MSL Cost Overrun Status and Plans. Available: http://www.lpi.usra.edu/pss/presentations/200810/greenMSL.pdf

[2] Mars Advanced Planning Group. 2006. Robotic Mars Exploration Strategy 2007-2016.

[3] Castano, R., Judd, M., Estlin, T., Anderson, R. C., Gaines, D., Castano, A., Bornstein, B., Stough, T., and Wagstaff, K. 2005. Current Results from a Rover Science Data Analysis System.

[4] Stancliff, S. B., Dolan, J., and Trebi-Ollennu, A. 2007. Planning to Fail Reliability as a Design Parameter for Planetary Rover Missions. In Proceedings of the 2007 Workshop on Measuring Performance and Intelligence of Intelligent Systems. PerMIS '07.

[5] Stancliff, S. B., Dolan, J., and Trebi-Ollennu, A. 2006. Mission Reliability Estimation for Multirobot Team Design. In Proceedings of the 2006 IEEE/RSJ International Conference on Intelligent Robots and Systems (Oct. 2006), pp. 2206 - 2211.

[6] Asikin, D. and Dolan, J. 2009. A Mission Taxonomy-Based Approach to Planetary Rover Cost-Reliability Tradeoff. In Proceedings of the 2009 Workshop on Performance Metrics for Intelligent Systems. PerMIS '09.

[7] NASA. 2006. Solar System Exploration. Available: http://www.lpi.usra.edu/vexag/road_map_final.pdf

[8] Mars Advance Planning Group. 2006. 2006 Update to Robotic Mars Exploration Strategy 2007 - 2016.

[9] Lagoudakis, M. G., et al. 2005. Auction-based Multi-robot Routing. In Proceedings of the Robotics Science and Systems Conference, pp. 343-350.

[10] NASA. 2004 - 2009. MER Rover Spirit Mission Log. Available: http://marsrovers.jpl.nasa.gov/mission/status_spirit.html

[11] NASA. 2003. NASA Facts: Mars Exploration Rover. Available: $\mathrm{http}: / /$ www.jpl.nasa.gov/news/fact_sheets/mars03rovers.pdf

[12] JPL-PACC. 2000. Presentation on Power Management in Past and Present JPL/NASA Missions (Sept. 26, 2000). Available from: $\mathrm{http} / / /$ newport.eecs.uci.edu/impacct/d_research/d_presentation/JPL-PAC C092600.ppt

[13] NASA. 2003. Mars Exploration Rover Launches Press Kit. Available: $\mathrm{http}: / /$ marsrover.nasa.gov/newsroom/merlaunch.pdf

[14] Leger, C., Trebi-Ollenu, A., Wright, J., Maxwell, S., Bonitz, B., Biesiadecki, J., Hartman, F., Cooper, B., Baumgartner, E., and Maimone, M. 2005. Mars Exploration Rover Surface Operations: Driving Spirit at Gusev Crater. 2005 IEEE Conference on Systems, Man, and Cybernetics.

[15] Dudek, G., Jenkin, M. R. M., Milios, E., and Wilkes, D. 1996. A Taxonomy for Multi-Agent Robotics. Autonomous Robots, vol. 3, no. 4, pp. $375-397$.

[16] Balch, T. 2002. Taxonomies of Multirobot Task and Reward. In Robot Teams: From Diversity to Polymorphism, Balch, T. and Parker, L. E., eds. Natick, MA: A K Peters.

[17] Yanco, H. A. and Drury, J. 2002. A Taxonomy for Human-Robot Interaction. In Proceedings of the AAAI Fall Symposium on Human-Robot Interaction, AAAI Technical Report FS-02-03, Falmouth, Massachusetts, November 2002, pp. $111-119$.

[18] Nehme, C., Crandall, J. W., and Cummings, M. L. 2007. An Operator Function Taxonomy for Unmanned Aerial Vehicle Missions. $12^{\text {th }}$ International Command and Control Research and Technology Symposium.

[19] Smith, T., Simmons, R., Singh, S., and Hershberger D. 2001. Future Directions in Multi-Robot Autonomy and Planetary Surface Construction. Proceedings of the 2001 Space Studies Institute Conference, May, 2001.

[20] Honeybee Robotics, Ltd. 2004. Robot Makes Historic Cut into Mars Rock. Available:

http://www.honeybeerobotics.com/press_releases/press_release_RAT_2. 8.04.pdf 